\title{
The Non-Equidistant Grey GRM $(1,1)$ Model and Its Application
}

\author{
Ruibiao Zou ${ }^{1}$, Zouxin Mou' ${ }^{2}$, Wei $\mathbf{Y i}^{2}$ \\ ${ }^{1}$ College of Sciences, Hunan Agriculture University, Changsha, China \\ ${ }^{2}$ Sany Heavy Industry Co. Ltd., Changde, China \\ Email: rbzou@163.com, yinanchang0@126.com
}

Received April 5, 2012; revised May 4, 2012; accepted May 15, 2012

\begin{abstract}
Applying the modeling method of Grey system and accumulated generating operation of reciprocal number for the problem of lower precision as well as lower adaptability in non-equidistant GM $(1,1)$ model, the calculation formulas were deduced and a non-equidistant GRM $(1,1)$ model generated by accumulated generating operation of reciprocal number was put forward .The grey GRM $(1,1)$ model can be used in non-equal interval \& equal interval time series and has the characteristic of high precision as well as high adaptability. Example validates the practicability and reliability of the proposed model.
\end{abstract}

Keywords: Background Value; Grey Model GRM $(1,1)$ Generated by Accumulated Generating Operation of Reciprocal Number; Non-Equal Interval; Accumulation Generation Operation; Grey System

\section{Introduction}

The grey model should be deemed as an important element of Grey System Theory. Since the Grey System Theory was firstly put forward by Professor Deng Julong, the grey model has been widely used in many fields [1]. Because of its research characteristics of "small sample" \& "poor information" and its advantages of simplicity \& practicability, the model GM $(1,1)$ has occupied an important position during the test data processing $\&$ testing and the online monitoring [1-6]. The grey system model is mostly based on the equidistance sequence; however, the original data obtained in the practical work is mostly based on the non-equidistant sequence; therefore, the establishment of non-equidistant sequence model has the certain practical and theoretical significances. For the non-negative discrete point range $\mathbf{X}^{(0)}$, its one-order accumulated generating sequence $\mathbf{X}^{(1)}$ should be monotonic; if the sequence is fitted by a curve $\mathbf{X}^{(1)}$, the curve should be also monotonic, so that the grey model GM $(1,1)$ can be forecasted. If $\mathbf{X}^{(0)}$ is decreased monotonically, due to the monotonic of $\mathbf{X}^{(1)}$, its value of model $\mathbf{X}^{(1)}$ should be monotonic; if $\mathbf{X}^{(1)}$ is used as the predictive value of accumulated decreasing generating reducing original sequence, there would be the unreasonable error of calculation. For the original sequence with the monotonic decreasing trend $\mathbf{X}^{(0)}$, the definition of reverse accumulated generating operation was put forward in the literature [7], so as to establish the grey model GOM (1,
1) based on the reverse accumulated generating operation; the definition of reciprocal generating operation was put forward in the literature [8], so as to establish the grey model GRM $(1,1)$ based on the reciprocal generating operation; the grey model GRM $(1,1)$ was improved in the literature [9], so as to establish the improved model-grey model CGRM $(1,1)$ based on the reciprocal generating operation. The generated sequence $\mathbf{X}^{(1)}$ with the monotonic decreasing trend can be use for the models established based on the reverse accumulated generating operation and the reciprocal generating operation; the value of model $\mathbf{X}^{(1)}$ of $\mathbf{X}^{(1)}$ can be gained after $\mathbf{X}^{(1)}$ is fitted by the monotonic curve; when the value of model is reduced as the predictive value $\mathbf{X}^{(0)}$ of $\mathbf{X}^{(0)}$, there would not be any unreasonable error of calculation produced by means of the modeling method as the same as the traditional accumulated and decreasing generating methods, so as to improve the modeling precision. In the literature [2], the spacing between sequences was used as the multiplier to establish the non- equidistance model GM $(1,1)$; this method could give tacit consent to the linear relationship between the data difference and the time difference; however, it was difficult to ensure whether the construction of model could be consistent with the actual condition. In the literature [3], the coefficient of standard deviation of sequence was decreased by means of the functional transformation method, so that the original sequence could be converted into the new data sequence, so as to estimate the model parameters, 
and then to establish the model GM $(1,1)$, resulting in the more complex calculation. In order to improve the fitting and prediction accuracy of mode GM $(1,1)$, the structure method of multi-background values was put forward in the literature [4-6], so as to establish a variety of non-equidistance models GM $(1,1)$. The improvement method of background value of non-equidis- tance model GM $(1,1)$ was put forward in the literature [4,5]; by means of this method, the one-order accumulated generating sequence could be fitted via the homogeneous exponential function, so as to obtain the higher accuracy; however, it could be seen from the form of albino differential equation solution of model GM $(1,1)$ that, the exponential form of one-order accumulated gen- erated sequence should not be deemed as the non-ho- mogeneous exponential form, but the homogeneous exponential form only after accumulated decreasing and reducing; if the one-order accumulated generating sequence was fitted via the homogeneous exponential func- tion, there would be the certain error of calculation. In the literature [6], the one-order accumulated generating sequence was fitted via the non-homogeneous exponential function to deduce the optimal background value calculation formula, so as to establish the non-equidis- tance model GM $(1,1)$. In this paper, the first component was used as the initial condition of differential equation of grey model; therefore, on the basis of the literature [8, 9], the non-equidistance model GM $(1,1)$ could be established based on the reciprocal accumulating generating method, which was characterized by the following: High accuracy; good theoretical and practical values. The pra- cticality and reliability of the established model could be shown in the fatigue test data processing examples.

\section{Non-Equidistance Model GRM $(1,1)$ Based on the Reciprocal Accumulated Generating Operation}

Definition 1. Supposing that the sequence is set as

$$
\mathbf{X}^{(00)}=\left[x^{(00)}\left(t_{1}\right), x^{(00)}\left(t_{2}\right), \cdots, x^{(00)}\left(t_{m}\right)\right],
$$

if $\Delta t_{i}=t_{i}-t_{i-1} \neq$ const $, i=2, \cdots, m$, then $\mathbf{X}^{(00)}$ should be referred to as the non-equidistance sequence. If

$$
x^{(0)}\left(t_{k}\right)=\frac{1}{x^{(00)}\left(t_{k}\right)} \quad(k=1,2, \cdots, m),
$$

then $\mathbf{X}^{(0)}=\left(x^{(0)}\left(t_{1}\right), x^{(0)}\left(t_{2}\right), \cdots, x^{(0)}\left(t_{m}\right)\right)$ should be referred to as the reciprocal sequence of $\mathbf{X}^{(00)}$.

Definition 2. Supposing that the sequence is set as

$$
\begin{gathered}
\mathbf{X}^{(1)}=\left[x^{(1)}\left(t_{1}\right), x^{(1)}\left(t_{2}\right), \cdots, x^{(1)}\left(t_{m}\right)\right], \\
x^{(0)}\left(t_{k}\right)=\frac{1}{x^{(00)}\left(t_{k}\right)} \quad(k=1,2, \cdots, m),
\end{gathered}
$$

if

$$
\begin{gathered}
x^{(1)}\left(t_{1}\right)=x^{(0)}\left(t_{1}\right), \\
x^{(1)}\left(t_{k+1}\right)=x^{(1)}\left(t_{k}\right)+x^{(0)}\left(t_{k+1}\right) \cdot \Delta t_{k+1}, k=1, \cdots, m-1,
\end{gathered}
$$

then $\mathbf{X}^{(1)}$ should be referred to as the first order reciprocal accumulated generating operation of non-equidistance sequence $\mathbf{X}^{(00)}$.

Definition 3. Supposing that the original data sequence is set as $\mathbf{X}^{(00)}=\left[x^{(00)}\left(t_{1}\right), x^{(00)}\left(t_{2}\right), \cdots, x^{(00)}\left(t_{m}\right)\right]$ (where, $x^{(00)}\left(t_{j}\right) \quad(j=1,2, \cdots, m)$ shall represent the observed value of variable quantity at the moment $t_{j}, m$ shall represent the number of data), then the sequence $\left[x^{(0)}\left(t_{1}\right), x^{(0)}\left(t_{2}\right), \cdots, x^{(0)}\left(t_{m}\right)\right]$ should be referred to as the non-equidistant sequence, i.e. the spacing $t_{j}-t_{j-1}$ should not be the constant.

In order to establish the model, the one-order reciprocal accumulated generating operation shall be carried out firstly for the original data, to generate a new sequence.

$$
\mathbf{X}^{(1)}=\left[x^{(1)}\left(t_{1}\right), x^{(1)}\left(t_{2}\right), \cdots, x^{(1)}\left(t_{m}\right)\right]
$$

where, $x^{(1)}\left(t_{j}\right) \quad(j=1,2, \cdots, m)$ can meet the Definition 2, i.e.

$$
x^{(1)}\left(t_{k}\right)= \begin{cases}x^{(0)}\left(t_{1}\right)+\sum_{j=1}^{k} x^{(1)}\left(t_{j}\right)\left(t_{j}-t_{j-1}\right) & (k=2, \cdots, m) \\ x^{(0)}\left(t_{1}\right) & (k=1)\end{cases}
$$

For $\mathbf{X}^{(1)}$ based on the one-order reciprocal accumulated generating operation, the non-equidistance model GRM $(1,1)$ should be established as per the first order differential equation set $\frac{\mathrm{d} x^{(1)}}{\mathrm{d} t}+a \zeta^{(1)}=b$ (in which $\zeta^{(1)}$ should be the background value), and its albino differential equation should be:

$$
\frac{\mathrm{d} x^{(1)}}{\mathrm{d} t}+a x^{(1)}=b
$$

Its differential form should be:

$$
x^{(0)}\left(t_{k}\right)+a z^{(1)}\left(t_{k}\right)=b
$$

In the formula

$$
z^{(1)}\left(t_{k}\right)=0.5 \cdot\left(x^{(1)}\left(t_{k}\right)+x^{(1)}\left(t_{k-1}\right)\right),
$$

$z^{(1)}\left(t_{k}\right)$ should be referred to as the background value of non-equidistance grey model GRM $(1,1)$ based on the reciprocal accumulated generating operation, i.e. the mean value of accumulated generating sequence.

If $\hat{\mathbf{a}}=[a, b]^{\mathrm{T}}$ is the parameter of non-equidistance model GRM $(1,1)$, then the least square of non-equidistance grey model GRM $(1,1)$ based on the reciprocal ac- 
cumulated generating operation should be estimated as:

$$
\hat{\mathbf{a}}=\left(\mathbf{B}^{\mathrm{T}} \mathbf{B}\right)^{-1} \mathbf{B}^{\mathrm{T}} \mathbf{Y}
$$

where,

$$
\mathbf{B}=\left[\begin{array}{cc}
-\frac{1}{2}\left(x^{(1)}\left(t_{2}\right)+x^{(1)}\left(t_{1}\right)\right) & 1 \\
-\frac{1}{2}\left(x^{(1)}\left(t_{3}\right)+x^{(1)}\left(t_{2}\right)\right) & 1 \\
\ldots & \ldots \\
-\frac{1}{2}\left(x^{(1)}\left(t_{m}\right)+x^{(1)}\left(t_{m-1}\right)\right) & 1
\end{array}\right], \quad \mathbf{Y}=\left[\begin{array}{c}
x^{(0)}\left(t_{2}\right) \\
x^{(0)}\left(t_{3}\right) \\
\cdots \\
x^{(0)}\left(t_{m}\right)
\end{array}\right],
$$

then the time response of differential Equation (4) of nonequidistance grey model should be:

$$
\hat{x}^{(1)}\left(t_{k}\right)=\frac{\hat{b}}{\hat{a}}+\left(x^{(0)}\left(t_{1}\right)-\frac{\hat{b}}{\hat{a}}\right) e^{-a\left(t_{k}-t_{1}\right)} \quad(k=1,2, \cdots, m)
$$

The fitted value of the reciprocal of original data gained after reduction should be

$$
\hat{x}^{(0)}\left(t_{k}\right)= \begin{cases}x^{(0)}\left(t_{1}\right), & (k=1) \\ \frac{\left(x^{(0)}\left(t_{1}\right)-\frac{\hat{b}}{\hat{a}}\right)\left(1-e^{a \Delta t_{k}}\right)}{\Delta t_{k}}, & (k=2,3, \cdots, m)\end{cases}
$$

The value of model of original sequence based on the Definition 1 should be $\hat{x}^{(00)}\left(t_{k}\right) \quad(k=1,2, \cdots, m)$

The absolute error of fitted data should be

$$
q\left(t_{k}\right)=\hat{x}^{(00)}\left(t_{k}\right)-x^{(00)}\left(t_{k}\right) .
$$

The relative error of fitted data (\%) should be

$$
e\left(t_{k}\right)=\frac{\hat{x}^{(00)}\left(t_{k}\right)-x^{(00)}\left(t_{k}\right)}{x^{(0)}(00)} \cdot 100 .
$$

The average value of relative errors of fitted data column should be

$$
\sum_{k=1}^{m}\left|e_{i}(k)\right| .
$$

After the analog values, predictive values, errors and other data can be gained, the non-equidistance model $\operatorname{GRM}(1,1)$ shall be inspected as well [1].

\section{Application Examples}

P. G. Forrest studied how the temperature would impact on the fatigue strength under the action of symmetrical cyclic load on many long-life materials. The experimental data on the fatigue strength of titanium alloy with the change of temperature should be shown at Table 1, which should be deemed as the non-equidistance se-
Table 1. Change relation of Ti alloy fatigue strength $\left(\sigma_{-1}\right)$ along with temperature $(\mathrm{T})$.

\begin{tabular}{cccc}
\hline$T$ & 100 & 130 & 170 \\
$\sigma_{-1}$ & 560 & 557.54 & 536.10 \\
$\mathrm{~T}$ & 210 & 240 & 270 \\
$\sigma_{-1}$ & 516.10 & 505.60 & 486.1 \\
$\mathrm{~T}$ & 310 & 340 & 380 \\
$\sigma_{-1}$ & 467.4 & 453.8 & 436.4 \\
\hline
\end{tabular}

quence. In this paper, the data indexed in the literature [2, 3 ] were applied, while the model was established by means of the method indexed in this paper, so as to obtain

$$
\begin{gathered}
a=-0.00098951, \quad b=0.0017647, \\
\hat{x}^{(1)}\left(t_{k}\right)=1.7852 e^{0.00098951(t-100)}-1.7834 .
\end{gathered}
$$

The fitted value of original data is:

$$
\begin{aligned}
\hat{\sigma}_{-1}= & {[560,557.7302,538.7297,517.8231,500.2108,} \\
& 485.5801,469.0376,453.0846,437.6491] .
\end{aligned}
$$

The absolute error of fitted data is:

$$
\begin{gathered}
q\left(t_{k}\right)=[0,-0.19022,-2.6297,-1.7231,5.3892, \\
0.51988,-1.6376,0.71544,-1.24913] .
\end{gathered}
$$

The relative error of fitted data (\%) is:

$$
\begin{gathered}
e\left(t_{k}\right)=[0,-0.034117,-0.49053,-0.33387,1.0659, \\
0.10695,-0.35037,0.15766,-0.28623] .
\end{gathered}
$$

The average value of relative errors of fitted data column is $0.31396 \%$.

In the literature [2], the original data was pre-processed with $\mathbf{t}=(\mathbf{T}-50) / 50, \quad \mathbf{X}^{(0)}=\left(\sigma_{-1}-400\right) / 50$, so that the maximum relative error of modeling was up to $4.86 \%$, and the average relative error of modeling was up to $3.19 \%$. In the literature [3], the model was established by means of the functional transformation method, so that the average relative error of modeling was up to $0.6587 \%$. In the literature [5], the one-order accumulated generating sequence was fitted via the homogeneous exponential function, so that the average relative error of modeling was up to $0.9765 \%$. As a result, it could be seen that there should be the adaptive and scientific methods indexed in this paper.

\section{Conclusion}

In this paper, applying the Grey System Theory with the first component of original data was used as the initial condition of grey differential equation, the non-equidistance model GRM $(1,1)$ based on the reciprocal accumulated generating operation was put forward; the MATLAB program was prepared. The model is suitable for both the modeling of equidistance sequence and the 
modeling of non-equidistance sequence with the characteristics of high precision, strong adaptability, etc. The correctness and validity of this model could be shown in the test data processing examples; due to its important practical and theoretical significances, this model should be widely used.

\section{REFERENCES}

[1] Y. X. Luo, L. T. Zhang and M. Li, "Grey Systems and Applications in Mechanical Engineering,” National University of Defense Technology Press, Changsha, 2001.

[2] Y. X. Luo and J. R. Zhou, “Non-Equidistance GM $(1,1)$ Model and Its Application in Fatigue Experimental Data Processing and On-Line Control," Journal of Mechanical Strength, Vol. 18, No. 3, 1996, pp. 60-63.

[3] Y. X. Luo, X. Wu and M. Li, "Function-Transfer Method of Parameters Estimation of Grey GM $(1,1)$ Model and Its Application,” Journal of Mechanical Strength, Vol. 24, No. 3, 2002, pp. 450-452.

[4] W. Z. Dai and J. F. Li, "Modeling Research on Non-
Equidistance GM $(1,1)$ Model,” Systems EngineeringTheory \& Practice, Vol. 25, No. 9, 2005, pp. 89-93.

[5] F. X. Wang, "Improvement on Unequal Interval Gray Forecast Model,” Fuzzy Information and Engineering, Vol. 6, No. 1, 2006, pp. 118-123.

[6] Y. M. Wang, Y. G. Dang, Z. X. Wang, "The Optimization of Background Value in Non-Equidistant GM $(1,1)$ Model," Chinese Journal of Management Science, Vol. 16, No. 4, 2008, pp. 159-162.

[7] Z. M. Song and J. L. Deng, "The Accumulated Generating Operation in Opposite Direction and Its Use in Grey Model GOM (1, 1)," Systems Engineering, Vol. 19, No. 2, 2001, pp. 66-69.

[8] B. H. Yang and Z. Q. Zhang, “The Grey Model Has Been Accumulated Generating Operation in Rcciprocal Number and Its Application," Mathematics in Practice and Theory, Vol. 33, No. 10, 2003, pp. 21-25.

[9] H. Zhou and X. G. Wang, “A Improvement of the Grey Model GRM $(1,1)$ Generated by Accumulattion Generating Operation of Reciprocal Number," Transactions of Shenyang Ligong University, Vol. 27, No. 4, 2008, pp. 84-86. 